\title{
Relation between postoperative antithrombin III concentrations and site of operation
}

\author{
SP GRAY, JA BILLINGS, VALERIE NEWTON, AUDREY OLIVE \\ From the Department of Biochemistry, Royal Naval Hospital, Haslar, Gosport, Hants PO12 $2 A A$
}

SUMMARY Antithrombin III concentrations were measured in 305 patients before and at intervals after operation for up to 12 days. The blood concentrations fell significantly after both major and minor surgery, but there was no relation between the expected degree of surgical trauma or the amount of blood transfused and the fall in concentration of antithrombin III. Some surgical procedures such as cholecystectomy, laparotomy, hernia repair, highly selective vagotomy, and transurethral resection of the prostate were associated with a greater depletion of antithrombin III than were other common operations and it is suggested that there are factors related to the actual site of the operation which affect the concentration of circulating antithrombin III in the postsurgical period.

Antithrombin IIJ is thought to be the major inhibitor of thrombin and activated factor $\mathrm{X}$ in the blood and has also been shown to act as a heparin co-factor, thereby enhancing its anticoagulant properties. ${ }^{1}$ Its relation to thromboembolic disease has been explored and it appears to play an important part in disseminated intra-vascular coagulation, pulmonary embolism, and deep venous thrombosis. ${ }^{1}$ Circulating concentrations of antithrombin III may be lowered in congenital deficiency of the protein $;^{1}$ after heparin treatment, ${ }^{2}$ in malnutrition, ${ }^{3}$ and in the postoperative phase. ${ }^{5}$ The risk of postoperative venous thrombosis emphasises the need for risk prediction and the assay of antithrombin III is currently being investigated as a potentially useful indicator of thromboembolism. ${ }^{6}$ ?

Surgery may initiate many responses associated with trauma such as in the complement, kallikreinkinin, coagulation and fibrinolytic pathways ${ }^{8}$ and one would expect major and minor surgery to elicit responses differing in type and extent. It may be feasible therefore to detect a relation between the extent of surgical injury associated with a particular type of operation and a component such as antithrombin III, which is involved in several of the pathways already mentioned. If such a relation was present, it could form the basis of a risk prediction for postoperative thromboembolism.

In this investigation antithrombin III activity was measured pre- and postoperatively on a group of

Accepted for publication 22 October 1980 patients undergoing a variety of surgery to establish the time-course and the activity in the postoperative period to see if any relation existed between the type of operation and the blood concentrations of antithrombin III.

\section{Patients and methods}

PATIENTS

Blood was collected from the following patients preand postoperatively at intervals for a period of up to 14 days (Table 1 ).

Table 1 Types of operation in 305 patients

\begin{tabular}{lc}
\hline Type of operation & No of patients \\
\hline Appendicectomy & 18 \\
Arterial (femoral-popliteal bypass) & 16 \\
Cholecystectomy & 60 \\
Colectomy and hemicolectomy & 17 \\
Fractures (no pre-trauma specimen) & 11 \\
Gastric: & 12 \\
selective vagotomy & 8 \\
partial gastrectomy & 23 \\
Gynaecology: & 14 \\
dilatation and currettage & 27 \\
repair & 15 \\
hysterectomy & 26 \\
Hernia & 9 \\
Laparotomy & 13 \\
Mastectomy & 11 \\
Pancreatic surgery & 11 \\
Thyroidectomy & 14 \\
Total hip replacement & 305 \\
Transurethral resection of prostate & \\
Total &
\end{tabular}


METHODS

Plasma was prepared by adding nine volumes of blood to one volume of $0.129 \mathrm{~mol} / 1$ of sodium citrate in a polystyrene tube and centrifuging at $\mathbf{2 0 0 0}$ $g$ for $20 \mathrm{~min}$ at $4{ }^{\circ} \mathrm{C}$. The plasma was then used for the measurement of antithrombin III by utilising the action of thrombin on a chromogenic substrate, S2238-that is, H-D-Phe-Pip-Arg-p-nitroanilide 2HC1 (KabiVitrum Ltd, London). All other reagents were taken from the Coatest Kit supplied by the manufacturers for the assay of antithrombin III. The method supplied by KabiVitrum was modified for use on an LKB 2086 Reaction Rate Analyser fitted with a 2082 Kinetic Data Processor (LKBSweden). The standard was a pooled lyophilised human plasma which was arbitrarily given the value of $100 \%$ antithrombin III.

Statistical methods such as the calculation of the mean and SD, Student's $t$ test and Wilcoxon test were taken from Colquhoun. ${ }^{9}$

\section{Results}

Antithrombin III concentrations after surgery followed a similar pattern in all the types of operation studied. From the preoperative level, values fell within a few days and then over the next week, rose above the initial preoperative level. A few days after this the concentrations returned to their initial state and remained there with very little fluctuation.

The fall in concentration was greater in some types of operation than in others and to illustrate this, two procedures-cholecystectomy and transurethral resection of the prostate were compared (Fig.). All values in the preoperative period were analysed statistically and the mean value was $100.58 \%$ antithrombin III activity (SD $\pm 10 \cdot 3)(n=305)$.

For each group of patients undergoing a particular type of surgery the mean value was calculated for $\%$ antithrombin III activity obtained in the first week after surgery. Mean values for each group are indicated in Table 2. Each postsurgical mean value was compared with the mean of presurgical antithrombin III activity from all of the patients using the Wilcoxon test in Mann-Whitney form and the significance of the difference calculated. The probability values are reported alongside the mean postoperation antithrombin III values in Table 2 .

Of the 305 patients investigated in the preoperative period, 14 had antithrombin III values below $80 \%$ normal activity-that is, approximately $5 \%$ of the total. The fall in antithrombin III activity was compared in the different types of operation and five groups differed significantly from the rest: cholecystectomy $p<0.05$; laparotomy $p<0.05$; repair of hernia $\mathrm{p}<0.05$; highly selective vagotomy

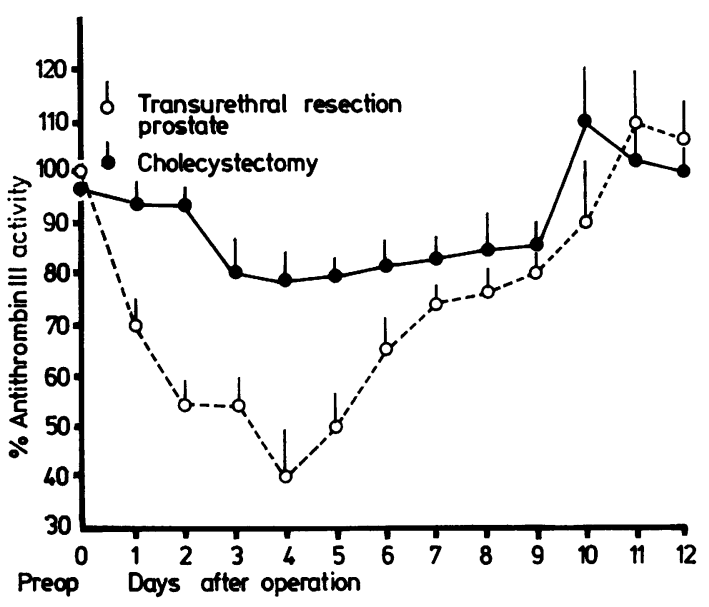

Relation between antithrombin III activities after surgery with time is shown. To illustrate the timecourse, two different types of operation were chosen; cholecystectomy (no of patients $=60$ ) and transurethral resection of the prostate (no of patients $=$ 14). Each point is the mean taken from all patients in that group and the vertical bars are standard errors of the mean (SEM).

Table 2 Mean antithrombin III values (\% activity) in the postoperative period

\begin{tabular}{lll}
\hline Operation & $\begin{array}{l}\text { Mean antithrombin } \\
\text { II (\%) }\end{array}$ & p value \\
& 101.0 & \\
\hline *Total hip replacement & 98.6 & NS \\
* Mastectomy & 96.3 & NS \\
*Pancreatic surgery & & \\
Gynaecology, dilatation and & 95.1 & $<0.05$ \\
currettage & 93.4 & $<0.05$ \\
Appendicectomy & 92.6 & NS \\
Fractures & 91.6 & $<0.05$ \\
*Colectomy/hemicolectomy & 90.9 & $<0.01$ \\
Hysterectomy & 87.9 & $<0.05$ \\
*Partial gastrectomy & 87.6 & $<0.001$ \\
Cholecystectomy & 87.2 & $<0.05$ \\
*Thyroid & 84.7 & $<0.001$ \\
*Arterial by-pass & 83.6 & $<0.001$ \\
Hernia & 82.2 & NS \\
Gynaecology: repair & 81.0 & $<0.001$ \\
Laparotomy & 80.9 & $<0.01$ \\
*Selective vagotomy & 60.6 & $<0.01$ \\
Transurethral resection prostate & & \\
\hline
\end{tabular}

NS = not significant.

*Blood transfusion was frequently necessary in these types of operation, while less so in the others. The fall in antithrombin III activity, however, did not differ significantly between these groups $(t=1.31$; df $=15$ ).

$p<0.025$; and transurethral resection of the prostate $\mathrm{p}<0.05$.

When those procedures in which blood transfusion was common were compared with those in which it was infrequent it was found that there was 
no significant difference in the postsurgical fall in antithrombin III activity ( $t$ test).

\section{Discussion}

Factors influencing the fall in the circulating concentration of antithrombin III in the postsurgical period are probably complex and difficult to elucidate but some of them can be evaluated from the data presented in this paper. Blood transfusion may be thought to be important after surgery perhaps as a supplement of the protein, but a comparison between operations in which blood is commonly given in the first week after operation and those in which transfusion is infrequent, does not bear out this supposition. There appears to be no detectable effect on postoperative antithrombin III concentrations in the blood. Similarly, antithrombin III activity is not particularly influenced by the degree of cellular and tissue trauma or duration of the operation. It is difficult also to isolate a particular target area such as abdominal surgery for instance which is consistently associated with depletion of antithrombin III concentrations.

A common factor, however, may be found in particular tissues which are rich in substances such as plasmin, ${ }^{10}$ which can neutralise antithrombin III. Examples are endothelial tissues, prostate, and uterus. Operations specifically on these tissues, or even on adjacent sites may then release fibrinolytic substances which in turn affect the activity of antithrombin III. If this release and subsequent fibrinolysis does occur, then potential thrombosis as a corollary of excessive thrombin may be prevented.
We are grateful to the Clinical Research Working Party who generously supported this work under Project No CRWP 9/77.

\section{References}

${ }^{1}$ Lane JL, Biggs R. The natural inhibitors of coagulation: antithrombin III, heparin co-factor and anti-factor Xa. In: Poller L, ed. Recent advances in blood coagulation. Vol 2. Edinburgh: Churchill Livingstone, 1977:123-39.

${ }^{2}$ Marciniak E, Cockerman JP. Heparin-induced decrease in circulating antithrombin III. Lancet 1977; ii:581-4.

3 Jimenez RA, Jimenez E, Ingram GIC, et al. Antithrombin activities in childhood malnutrition. J Clin Pathol 1979; 32:1025-9.

${ }^{4}$ Stathakis N, Papayannis AG, Gardikas CD. Postoperative antithrombin III concentration. Lancet $1973 ; \mathrm{i}: 430$.

${ }^{5}$ Abert M, Nilsson IM, Hedner U. Antithrombin III after operation. Lancet 1973 ;ii:1337.

- Sculley MF, Kakkar VV. Methods for semi-micro and automated determination of thrombin, antithrombin and heparin co-factor using the substrate H-D-Phe-PipArg-p-nitroanilide 2HCl. Clin Chim Acta 1977;79:595602.

${ }^{7}$ Chan V, Chan TK, Wong V, Tso SC, Todd D. Determination of antithrombin III by radioimmunoassay and its clinical application. Br J Haematol 1979;41:363-72.

${ }^{8}$ Seegers WH. Antithrombin III. Theory and Clinical Applications. Am J Clin Pathol 1978;69:367-71.

- Colquhoun D. Lectures on Biostatistics. Oxford: Clarendon Press, 1971.

${ }^{10}$ Kernoff PBA, McNicol GP. Normal and abnormal fibrinolysis. $\mathrm{Br}$ Med Bull 1977;33:239-43.

Requests for reprints to: Dr SP Gray, Department of Biochemistry, Royal Naval Hospital, Haslar, Gosport, Hants PO12 2AA, England. 